\title{
Notificação de Parceiros - Caracterização da População e Preferências de 100 Doentes Consecutivos da Consulta de Venereologia de um Hospital Português
}

\author{
Alexandre Miroux-Catarino', João Borges-Costa² \\ 'Médico Interno de Dermatovenereologia/Resident of Dermatology and Venereology, Serviço de Dermatologia, Centro Hospitalar \\ de Lisboa Ocidental, Lisboa, Portugal \\ ${ }^{2}$ Assistente Hospitalar de Dermatovenereologia / Consultant, Dermatology and Venereology, Centro Hospitalar Lisboa Norte; \\ Professor Auxiliar Convidado de Dermatologia e Venereologia / Associated Professor, Dermatology and Venereology, Faculdade de \\ Medicina da Universidade de Lisboa; Professor Auxiliar Convidado de Microbiologia / Professor, Microbiology, Instituto de Higiene \\ e Medicina Tropical da Universidade Nova de Lisboa, Lisboa, Portugal
}

\begin{abstract}
RESUMO - Introdução: A notificação de parceiros tem um papel fundamental no controlo epidemiológico das infeções sexualmente transmissíveis (IST) que apresentam incidências crescentes. Material e Métodos: Foi realizado um questionário a 100 doentes consecutivos da consulta de Venereologia do Hospital Santa Maria em Lisboa referente à notificação dos parceiros. Resultados: Na população estudada, 43 tinham história de IST anterior, 25 dos quais tinham o diagnóstico prévio de infeção $\mathrm{VIH}$. Sessenta e quatro doentes disseram ter informado os parceiros sobre a IST diagnosticada. Dos que não avisaram $50 \%$ não o fez por já não ter contacto com os parceiros e $36 \%$ não achou importante. Em relação aos métodos de notificação de parceiros: $98 \%$ achou adequado levar um folheto informativo sobre a doença aos parceiros; $72 \%$ concordaria em entregar uma autorização de consulta ao parceiro, $58 \%$ considerou que levar diretamente a medicação seria uma opção, $58 \%$ achava adequado que o médico de família do parceiro o convocasse para uma consulta, e $42 \%$ considerou adequado o envio de e-mail ou mensagem de telemóvel. Discussão: A elevada percentagem de diagnósticos prévios aponta para um conjunto de indivíduos pertencentes aos grupos com maior risco de transmissão de IST. Uma percentagem elevada $(36 \%)$ dos que não informaram os parceiros acharam que não seria importante, o que pode revelar uma falha de comunicação do profissional de saúde. O método considerado mais vezes adequado foi o da notificação passiva. Conclusão: É necessário tornar a notificação dos parceiros mais eficaz como forma de reduzir a incidência das IST.

PALAVRAS-CHAVE - Comportamento Sexual; Doenças Sexualmente Transmissíveis; Encaminhamento e Consulta; Parceiros Sexuais.
\end{abstract}

\section{Partner Notification - Characterization of the Population and Preferences of 100 Consecutive Patients of the Venereology Consultation in a Portuguese Hospital}

ABSTRACT - Introduction: Partner notification plays a key role in the epidemiological control of the increasingly frequent sexually transmitted infections (STI). Material and Methods: One hundred consecutive patients from the Venereology consultation of the Santa Maria Hospital in Lisbon answered a questionnaire on notification of partners. Results: Forty three of the individuals had a previous history of STI, 25 of which had a previous diagnosis of HIV infection. Sixty-four patients said that they informed their partners about the diagnosis. Of those who did not inform, 50\% no longer have contact with the partners

Correspondência: Alexandre Miroux-Catarino Serviço de Dermatologia - Hospital Egas Moniz Rua R. da Junqueira 126

1349-019 Lisboa, Portugal

E-mail: alexandre_catarino@hotmail.com

DOI: https://dx.doi.org/10.29021/spdv.76.2887
Recebido/Received

17 Janeiro/January 2018

Aceite/Accepted

27 Março/March 2018 
and $36 \%$ consider that was not important. Regarding partner notification methods: $98 \%$ found appropriate to bring a leaflet about the disease to the partners; $72 \%$ would agree to give a consultation authorization to the partner, $58 \%$ considered that taking the medication directly to the partner would be an option, $58 \%$ thought appropriate that the partner's family doctor call them for a consultation; and $42 \%$ considered appropriate to send an email or mobile message. Discussion: The high percentage a previous STI diagnosis suggests this is a group of individuals with the highest risk of STI transmission. A high percentage $(36 \%)$ of those who did not inform the partners felt that it would not be important, which may reveal a failure of communication from the health professional. The method considered the most suitable was passive reporting. Conclusion: It is necessary to improve effective partner notification at it is highly needed to reduce the incidence of STI.

KEYWORDS - Referral and Consultation; Sexual Behavior; Sexual Partners; Sexually Transmitted Diseases.

\section{INTRODUÇÃO}

A incidência de infeções sexualmente transmissíveis (IST) tem aumentado na Europa nas últimas duas décadas. $^{1}$

A notificação de parceiros é o processo pela qual os parceiros sexuais de determinado indivíduo diagnosticado com uma IST (indivíduo índex) são informados da sua exposição a determinada infeção e da necessidade de avaliação e possível tratamento. ${ }^{2}$ No controlo das IST o tratamento dos parceiros é essencial para interromper a cadeia de transmissão, evitar a reinfeção e impedir o desenvolvimento de complicações resultantes de infeções crónicas assintomáticas. ${ }^{3,4}$

Apesar do maior conhecimento populacional sobre as IST, verifica-se que os comportamentos de risco se mantêm e a notificação dos parceiros assume resultados pouco satisfatórios contribuindo para isso o estigma associado às IST. ${ }^{5-7}$ Por outro lado, mesmo quando os contactantes são notificados nem sempre estes procuram os cuidados de saúde. Os estudos apontam para uma taxa de apenas $40 \%$ a $60 \%$ de parceiros que procuram os cuidados de saúde. ${ }^{8} \mathrm{Um}$ estudo realizado em Portugal em indivíduos diagnosticados com Chlamydia trachomatis demonstrou uma taxa de notificação pelos indivíduos índex de 34\%, e verificou que a referênciação era mais provável em indivíduos heterossexuais e com menor número de parceiros nos 6 meses anteriores. ${ }^{9}$ A associação entre relações estáveis e menor número de parceiros com maior taxas de notificação é corroborada por outros estudos. ${ }^{10}$

Existem quatro formas principais de tratamento e notificação de parceiros: 1. Notificação passiva: o indivíduo diagnosticado informa os parceiros; pode ser simples ou "incentivada" se incluir material de apoio como folhetos informativos; 2 . Medicação entregue pelo parceiro: o indivíduo índex leva diretamente a medicação aos contactantes; 3. Notificação pelo profissional de saúde: os serviços de saúde contactam os parceiros após o indivíduo index referir quem são; 4 . Notificação contratual: os serviços de saúde contactam os parceiros se até uma data estipulada os parceiros não recorreram à consulta por indicação do indivíduo diagnosticado."

A notificação de parceiros como aspeto importante no controlo de transmissão de IST data do início do século $X X .^{12-14} \bigcirc$ método de notificação e o seu contexto legal variam substancialmente entre os países da Europa. Esta elevada variabilidade no método de notificação prende-se em parte com a ausência de evidência robusta que suporte um método em detrimento de outros. ${ }^{8,11}$ A Organização Mundial de Saúde recomenda que a notificação seja realizada numa base voluntária, no entanto a notificação de parceiros é obrigatória em vários países da Europa. ${ }^{15,16}$ A Suécia é o país da Europa com maior regulamentação sendo que a notificação é obrigatória para os profissionais de saúde, $\mathrm{o}$ indivíduo index deve aceitá-la e os parceiros notificados têm de aceitar a realização de eventuais exames complementares de diagnóstico e tratamento. $\mathrm{Na}$ Suécia esta obrigação é válida para VIH, sífilis, Neisseria gonorrhoea, Chlamydia trachomatis, hepatite B e hepatite C. A criminalização da transmissão do VIH ou outras IST é outro ponto importante. A criminalização significa que a lei condena especificamente indivíduos que sabendo que estão infetados com uma IST não informam o parceiro e o expoêm ao risco de infeção. Essa criminalização pode ter o efeito contraproducente de levar a que os indivíduos se recusem a realizar o rastreio de IST, nomeadamente do $\mathrm{VIH}$. Na Europa, em nove países (Áustria, Bélgica, Dinamarca, Finlândia, Grécia, Malta, Noruega, Suécia e Reino Unido) há leis que criminalizam a transmissão de IST, em quatro deles apenas para $\circ \mathrm{VIH} .{ }^{17}$

Em Portugal, a notificação dos parceiros é voluntária e não existem leis que obriguem a proceder à notificação. Ainda, não há legislação específica sobre criminalização da transmissão de IST, no entanto há casos de condenação por transmissão de $\mathrm{VIH} .{ }^{17}$

O objetivo é avaliar a taxa de notificação de parceiros em indivíduos seguidos numa consulta de Venereologia num hospital de referência em Lisboa e perceber se existe uma forma de notificação preferida pelos doentes.

\section{MÉTODOS}

Foi realizado um questionário nos meses de Abril e Maio de 2017 a 100 doentes consecutivos da consulta de Venereologia do Hospital Santa Maria - Centro Hospitalar de Lisboa Norte. Foram incluídos doentes observados pela primeira vez ou em consultas subsequentes. Os doentes avaliados em primeira consulta já traziam um diagnóstico de IST, por exemplo da urgência de dermatologia do Hospital Santa Maria, pelo que era possível perguntar se nesse 
Tabela 1 - Diagnósticos atuais dos doentes em estudo.

\begin{tabular}{l|c}
\multicolumn{1}{c|}{ DIAGNÓSTICO } & Número de casos \\
\hline Condilomas acuminados & 40 \\
\hline Sífilis & 19 \\
\hline Herpes Genital & 18 \\
\hline Molusco contagioso & 5 \\
\hline Infeção VIH & 4 \\
\hline Infeção por Neisseria gonorrhoea & 4 \\
\hline Infeção por Chlamydia trachomatis & 4 \\
\hline Uretrites (sexo masculino) 1 & 2 \\
\hline
\end{tabular}

Três indivíduos incluídos no estudo foram enviados à consulta com o diagnóstico de IST que não se confirmou.

intervalo de tempo os parceiros tinham sido avisados. $\bigcirc$ questionário era realizado de forma oral aos doentes e o formulário preenchido pelo médico, sem registo de dados pessoais, após o consentimento oral do doente.

Esse questionário compreendia: idade, sexo, orientação sexual, etnia, país, IST prévias e se os parceiros tinham sido avisados aquando do diagnóstico, IST atual, número de parceiros nos últimos seis meses, se os parceiros foram avisados ou não e porquê, e quais os meios considerados adequados como forma de notificação.

\section{RESULTADOS}

Os diagnósticos atuais dos 100 doentes do estudo estão discriminados na Tabela 1 .

A média de idades dos indivíduos que responderam ao questionário foi de 39 anos, com um intervalo de 16 a 74 anos. A população questionada era composta por 73 indivíduos do sexo masculino e 27 do sexo feminino, 81 caucasianos vs 19 não caucasianos, 78 portugueses e 22 não portugueses. Em relação à orientação sexual 72 indivíduos eram heterossexuais e 28 homossexuais. Os indivíduos homossexuais eram todos do sexo masculino, pelo que cerca de $38 \%$ dos homens eram homens que têm sexo com homens (HSH). 43 tinham história de IST anterior, 28 dos quais uma IST, 12 tinham história de duas IST e 3 de três IST anteriores. Vinte e cinco indivíduos tinham o diagnóstico prévio de infeção pelo $\mathrm{VIH}$.

Dos que tinham IST anteriores, $65 \%$ referiu que avisou os parceiros versus 35\% que não tinha avisado os parceiros no momento dos diagnósticos anteriores.

Em relação ao número de parceiros sexuais nos últimos 6 meses: 5 indivíduos - 0 parceiros; 59 indivíduos - 1 parceiro, 31 indivíduos - 2 a 5 parceiros, e 3 indivíduos $->5$ parceiros. Em média os indivíduos heterossexuais tiveram
1,4 parceiros nos útimos 6 meses em comparação com uma média de 2,8 nos homossexuais.

Sessenta e quatro doentes (64\%) referiam ter informado os parceiros sobre a IST diagnosticada e 36 não o terão feito. Entre os indivíduos do sexo masculino, 67\% dos heterossexuais e $52 \%$ dos $\mathrm{HSH}$ referiu ter avisado. Dos indivíduos sem diagnóstico prévio de IST, 72\% referiam ter informado os parceiros, em comparação com $54 \%$ dos indivíduos que tinham diagnóstico anterior de IST. Entre os indivíduos que não tinham informado os parceiros aquando do diagnóstico prévio, apenas $27 \%$ referiam ter avisado os parceiros do diagnóstico atual de IST. Dos 36 que disseram não ter avisado, 50\% não o fez por já não ter contacto com os parceiros, $36 \%$ não achou importante, $5,6 \%$ tiveram receio da reação, 5,6\% referiam como motivo a ausência de sintomatologia no parceiro e 2,7\% (um caso) não quis informar. Quem avisou os parceiros em média teve 1,4 parceiros nos 6 meses anteriores, face a uma média de 2,4 parceiros de quem não avisou.

Questionou-se a adequação de possíveis métodos de notificação de parceiros e os resultados estão discriminados na Tabela 2.

\section{DISCUSSÃO}

Os objetivos da notificação de parceiros são a quebra das cadeias de transmissão na comunidade e a redução das taxas de infeção/reinfeção. É assim fundamental perceber se os métodos de notificação são eficazes e conhecer os métodos preferidos pela população. A notificação de parceiros é difícil pois existe um estigma associado às IST, frequentemente associado a medo de reação do parceiro, vergonha ou sentimento de culpa. ${ }^{18,19}$

$\mathrm{Na}$ população que respondeu ao questionário destaca-se o predomínio do sexo masculino, caucasianos e 
Tabela 2 - Modos de notificação de parceiros considerados adequados pelos indivíduos com IST nos questionários.

\begin{tabular}{l|c}
\hline \multicolumn{1}{|c|}{ MODO DE NOTIFICAÇÃO } & $\begin{array}{c}\text { Percentagem de } \\
\text { indivíduos que considerou } \\
\text { opção adequada }\end{array}$ \\
\hline Levar um folheto informativo sobre a doença ao parceiro & $98 \%$ \\
\hline Entregar uma autorização de consulta ao parceiro & $72 \%$ \\
\hline Levar diretamente a medicação & $58 \%$ \\
\hline Convocar o parceiro pelo Médico de Família & $58 \%$ \\
\hline Enviar email ou mensagem por telemóvel ao parceiro pelos serviços de saúde & $42 \%$ \\
\hline
\end{tabular}

portugueses e há também uma percentagem elevada de homens que têm sexo com homens. Verifica-se que $43 \%$ dos indivíduos já tinham diagnósticos prévios de IST, alguns deles com mais do que um diagnóstico prévio, e $25 \%$ com diagnóstico prévio conhecido de infeção $\mathrm{VIH}$. Estes valores elevados demonstram uma percentagem relevante de indivíduos pertencentes aos grupos com maior risco de transmissão de IST com comportamentos sexuais que contribuem desproporcionalmente para a transmissão das IST, nomeadamente pela não notificação de parceiros verificada nestes casos. Verificou-se também uma média superior de parceiros nos indivíduos homossexuais, já descrita por outros autores. ${ }^{20}$

O questionário era realizado pelo médico o que pode contribuir para viéses de resposta dos indivíduos questionados. Dos indivíduos questionados $64 \%$ referiu ter informado os parceiros, valor elevado se compararmos com os $34 \%$ de contactantes que procuraram os cuidados de saúde encontrados no estudo de I Santo et al. ${ }^{9}$ Uma vez que não foram contactados os parceiros não é possível aferir a veracidade deste valor, associado ao possível viés referido anteriormente. Uma percentagem superior de homens heterosexuais (67\% vs 52\%) referiu ter avisado os parceiros, o que segue o encontrado no estudo de I Santo et al em que os homens heterossexuais referenciaram 1,4 vezes mais que os $\mathrm{HSH}$, apesar de não ter sido uma diferença estatisticamente significativa. ${ }^{9}$

Trinta e quatro indivíduos responderam que não tinham informado os contactos sexuais, e em $50 \%$ dos casos devia-se a já não terem contacto com os parceiros. Verifica-se que em média os indivíduos que não avisaram os parceiros tinham maior número de parceiros nos últimos 6 meses o que pode estar a associado a comportamentos e práticas sexuais distintas e ausência de relações estáveis como apontam vários estudos.9,10 Não se podem também excluir também falhas na comunicação do profissional de saúde, no momento do diagnóstico, sobre a importância dessa mesma notificação, ${ }^{21}$ uma vez que $36 \%$ dos indivíduos que não informaram os parceiros referiram que pensavam não ser relevante avisar.

$\mathrm{Na}$ escolha dos métodos preferenciais para notificação, a notificação pelo próprio indivíduo com o apoio de folheto informativo foi o método mais escolhido com 98\% dos indivíduos a achar adequado. Esta preferência está de acordo com outros estudos. ${ }^{19,22,23} \mathrm{Um}$ estudo que comparava entre a notificação pelo parceiro e formas de notificação pelos serviços de saúde por carta, e-mail ou mensagem de telemóvel, constatou que a preferência era de que fossem informados pelos próprios parceiros. $65,8 \%$ dos questionados considerava um bom método serem avisados pelo parceiro, face a taxas de aprovação inferiores a $50 \%$ para os outros métodos. Por outro lado, os indivíduos preferiam ser informados de que teriam de entrar em contacto com a clínica em oposição a serem informados de que poderiam ter contraído uma IST (31,8\% vs $17 \%$ no caso das mensagens de telemóvel. ${ }^{23} \mathrm{Um}$ estudo realizado em adolescentes também demonstrou que a notificação pessoal era o método preferido nesta faixa etária. ${ }^{19} \mathrm{~A}$ existência de um folheto informativo com referência à potencial IST a que um parceiro teve contacto parece aumentar o número de parceiros que recorre aos sistemas de saúde como verificado num estudo realizado em Londres em infeções por Chlamydia trachomatis (84\% vs 33\%). ${ }^{24}$

A possibilidade de entregar uma autorização de consulta ao parceiro foi um método considerado relevante para $78 \%$ dos inquiridos dando relevo à facilidade de acesso aos cuidados de saúde como forma de tratar os parceiros. O envio de e-mail ou mensagem de texto por telemóvel foi o método que menos agradou aos questionados no entanto deverá ser mais um contributo a ter em conta que poderá ajudar em alguns grupos de maior risco.

A possibilidade de o indivíduo index levar a medicação foi considerado adequado por $58 \%$ dos indivíduos, no entanto parece ser um método eficaz na prevenção da re-infeção do indivíduo e resultar em maior número de parceiros tratados, pelo que deve ser fomentada nos casos em que é adequada, embora não permita o rastreio de outras IST nos parceiros. ${ }^{11}$ 


\section{CONCLUSÃO}

A notificação de parceiros é uma das armas mais importantes no controlo das infeções sexualmente transmissíveis e é fundamental que seja compreendida pelos doentes e otimizada a adesão à mesma. As várias formas de notificação devem ser comparadas em termos de eficácia e custos e adequadas aos diferentes indivíduos, grupos de risco ou sociedades em questão.

Conflitos de interesse: Os autores declaram não possuir conflitos de interesse.

Suporte financeiro: $O$ presente trabalho não foi suportado por nenhum subsídio ou bolsa.

Confidencialidade dos dados: Os autores declaram ter seguido os protocolos do seu centro de trabalho acerca da publicação dos dados de doentes.

Protecção de pessoas e animais: Os autores declaram que os procedimentos seguidos estavam de acordo com os regulamentos estabelecidos pelos responsáveis da Comissão de Investigação Clínica e Ética e de acordo com a Declaração de Helsínquia da Associação Médica Mundial

Conflicts of interest: The authors have no conflicts of interest to declare.

Financing Support: This work has not received any contribution, grant or scholarship.

Confidentiality of data: The authors declare that they have followed the protocols of their work center on the publication of data from patients.

Protection of human and animal subjects: The authors declare that the procedures followed were in accordance with the regulations of the relevant clinical research ethics committee and with those of the Code of Ethics of the World Medical Association (Declaration of Helsinki).

\section{REFERÊNCIAS}

1. European Centre for Disease Prevention and Control. Sexually transmitted infections in Europe 2013. Stockholm: ECDC; 2015.

2. Ward H, Bell G. Partner notification. Medicine. 2014;42:314-7.

3. Althaus $\mathrm{CL}$, Turner $\mathrm{KM}$, Mercer $\mathrm{CH}$, Auguste $\mathrm{P}$, Roberts $T E$, Bell $G$, et al. Effectiveness and cost-effectiveness of traditional and new partner notification technologies for curable sexually transmitted infections: observational study, systematic reviews and mathematical modelling. Health Technol Assess. 2014;18:1-100, vii-viii.

4. Hogben M. Partner notification for sexually transmitted diseases. Clin Infect Dis. 2007;44 Suppl 3:S16074.

5. van Aar F, Schreuder I, van Weert Y, Spijker R, Gotz $\mathrm{H}, \mathrm{Op}$ de Coul E. Current practices of partner notification among MSM with HIV, gonorrhoea and syphilis in the Netherlands: an urgent need for improvement. BMC Infect Dis. 2012;12:114.

6. Arunkumar S, Kamoji SG, Kasatti G. Partner notification and treatment Institute of Venereology-experience.
Indian J Sex Transm Dis. 2013;34:10-3.

7. Low N, Broutet N, Adu-Sarkodie Y, Barton P, Hossain $M$, Hawkes S. Global control of sexually transmitted infections. Lancet. 2006;368:2001-16.

8. Trelle S, Shang A, Nartey L, Cassell JA, Low N. Improved effectiveness of partner notification for patients with sexually transmitted infections: systematic review. BMJ. 2007;334:354.

9. Santo I, Azevedo J, Nunes B, Gomes JP, Borrego MJ. Partner notification for chlamydia trachomatis urogenital infections: eight years of patient referral experience in the major Portuguese sexually transmitted infections clinic, 2000-07. Int J STD AIDS. $2011 ; 22: 548-51$.

10. Shumin $C$, Zhongwei $L$, Bing $L$, Rongtao $Z$, Benqing $S$, Shengii Z. Effectiveness of self-referral for male patients with urethral discharge attending a sexually transmitted disease clinic in China. Sex Transm Dis. 2004;31:26-32.

11. Ferreira A, Young T, Mathews C, Zunza M, Low N. Strategies for partner notification for sexually transmitted infections, including HIV. Cochrane Database Syst Rev. 2013:Cd002843.

12. Harrison LW. Anti-Venereal Measures in Denmark and Sweden. Br J Vener Dis. 1939;15:1-17.

13. Wertheimer WJ. The politics of STDs. Dwindling resources for a growing problem. Prim Care. 1990;17:18394.

14. Cowan FM, French R, Johnson AM. The role and effectiveness of partner notification in STD control: a review. Genitourin Med. 1996;72:247-52.

15. Laar AK, DeBruin DA, Craddock S. Partner notification in the context of HIV: an interest-analysis. AIDS Res Ther. 2015;12:15.

16. Lowndes CM, Fenton KA. Surveillance systems for STIs in the European Union: facing a changing epidemiology. Sex Transm Infect. 2004;80:264-71.

17. European Centre for Disease Prevention and Control.Public health benefits of partner notification for sexually transmitted infections and HIV. Stockholm: ECDC; 2013.

18. Cavalcante EG, Miranda MC, Carvalho AZ, Lima IC, Galvao MT. Partner notification for sexually transmitted infections and perception of notified partners. Rev Esc Enferm USP. 2016;50:450-7.

19. Reed JL, Huppert JS, Gillespie GL, Taylor RG, Holland CK, Alessandrini EA, et al. Adolescent patient preferences surrounding partner notification and treatment for sexually transmitted infections. Acad Emerg Med. 2015;22:61-6.

20. van Aar F, van Weert Y, Spijker R, Gotz H, Op de Coul E. Partner notification among men who have sex with men and heterosexuals with STI/HIV: different outcomes and challenges. Int J STD AIDS. 2015;26:56573.

21. Op de Coul EL, Spijker R, van Aar F, van Weert Y, 
de Bruin M. With whom did you have sex? Evaluation of a partner notification training for STI professionals using motivational interviewing. Patient Educ Couns. 2013;93:596-603.

22. Kamanga G, Brown L, Jawati P, Chiwanda D, Nyirenda N. Maximizing HIV partner notification opportunities for index patients and their sexual partners in Malawi. Malawi Med J. 2015;27:140-4.
23. Apoola A, Radcliffe KW, Das S, Robshaw V, Gilleran G, Kumari BS, et al. Patient preferences for partner notification. Sex Transm Infect. 2006;82:327-9.

24. Wright A, Chippindale S, Mercey D. Investigation into the acceptability and effectiveness of a new contact slip in the management of Chlamydia trachomatis at a London genitourinary medicine clinic. Sexually Transmitted Infections. 2002;78:422-4. 